\title{
EFECTIVIDAD ANÉSTESICA DEL PARCHE OUTDOL PARA EL TRATAMIENTO DE DETARTRAJE
}

PARIONA-MINAYA MARIA *

Recibido: 16/11/2015

Aceptado: 20/12/2015

\section{RESUMEN}

El objetivo del presente estudio fue verificar la efectividad del parche OUTDOL, en la anestesia de mucosa gingival. Se aplicó a 28 pacientes que sirvieron como grupo experimental y grupo control a la vez sectorizando la arcada dentaria en una hemiarcada experimental y otra hemiarcada control. Se aplicó el parche por espacio de 5 minutos y se procedió a realizar el detartraje, con las características usuales. Se aplicó un cuestionario de evaluación del dolor (escala visual analógica EVA) luego del procedimiento de detartraje, obteniendo un valor por cada pieza dentaria y se procedió a promediar el conjunto de los valores en cada hemiarcada. Los resultados obtenidos fueron que el promedio de dolor para el lado control fue de 0.95 con desviación estándar de 0.39; mientras que el dolor en la zona del parche fue de 0.29 con desviación estándar de 0.39 , en una escala de 0 a 5 . La hipótesis, fue comprobada mediante la prueba estadística T de Student $(p<0.001)$ demostrando que hay diferencia estadísticamente significativa entre los valores de dolor en la zona sin parche comparando con la zona con parche, lo que demostró la efectividad del parche OUTDOL.

Palabras Clave. Dolor, anestesia, encía, parche.

\section{ABSTRACT}

The aim of this study was to verify the effectiveness of the OUTDOL patch in anesthetizing gingival tissue. This was applied to 28 patients who served both as experimental and control group. The dental arch was divided into two zones in order to have one half as experimental group and the other half as control group. The patch was applied for 5 minutes and then the tartar was removed following usual procedures. A pain evaluation questionnaire (VAS visual analogue scale) was applied after the tartar removal, obtaining a value for each dental piece and proceeding to average all the values for each middle arch. According to the results, the control arch registered an average pain of 0.95 with a standard deviation of 0.39 ; whereas the pain registered on the patch zone was 0.29 with a standard deviation of 0.39 on a scale of 0 to 5 . The hypothesis was tested through the student T-test statistics ( $p<0.001)$, demonstrating that there is significant statistical difference between the pain values on the unpatched area compared to those on the OUTDOL patch area. It checked the effectiveness of the patch OUTDOL.

Keywords. Pain, Anesthesia, gingiva, patch.

\section{INTRODUCCIÓN}

El dolor en los procedimientos dentales, es una gran barrera que los pacientes deben enfrentar al momento de tomar la decisión de acudir a la consulta, motivo por el cual, es necesario que el odontólogo desarrolle procedimientos, menos invasivos y menos dolorosos, la presente investigación probó la efectividad de un parche anestésico en pacientes del área de periodoncia. "Los anestésicos locales son medicamentos que bloquean en forma reversible la conducción del impulso nervioso pues inhiben la excitación del nervio en las fibras mielínicas y no mielínicas" 1 . "Los anestésicos locales se ligan reversiblemente a un receptor específico dentro del poro de los canales de sodio en nervios y bloquean los desplazamientos de iones en dichos orificios. Al ser aplicados localmente al tejido nervioso en concentraciones apropiadas, dichos anestésicos actúan en cualquier parte del sistema nervioso y en todos los tipos de fibras al bloquear de manera reversible los potenciales de acción que sustentan la conducción nerviosa"2. "Los anestésicos locales son capaces de bloquear la conducción nerviosa en forma selectiva, reversible y temporaria, cuando se aplican a zonas restringidas del organismo y sin afectar otros tejidos"3. "Los anestésicos locales bloquean la conducción al disminuir o prevenir el gran incremento transitorio en la permeabilidad de las membranas excitables al $\mathrm{Na}+$ que normalmente se produce por una despolarización leve de la membrana. Esta acción de los anestésicos locales se debe a su interacción directa con canales de $\mathrm{Na}+$ de compuerta de voltaje"4. "Se piensa que los anestésicos locales actúan por estabilización de la membrana celular y previenen el movimiento hacia adentro de los iones de sodio. La mayor parte de los anestésicos locales son amidas terciarias(R) que se administran como clorhidratos solubles en agua (R.HCL). Después de la inyección la amina libre es liberada debido al pH alcalino de fluido tisular. La amina libre $(R)$ se difunde a través de la vaina nerviosa y el tejido periaxonal, y atrae a los iones de hidrogeno $(\mathrm{H}+), \mathrm{RH}+$ es la forma más activa del anestésico local. Se piensa que las moléculas de $\mathrm{RH}+$ se combinan dentro de los canales de sodio en la membrana nerviosa, que se abren durante la despolarización

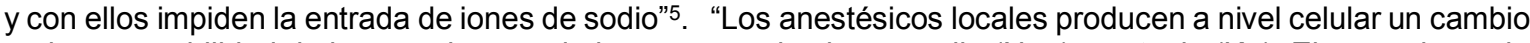
en la permeabilidad de las membranas de la neurona a los iones sodio $(\mathrm{Na}+)$ y potasio $(\mathrm{K}+)$. El mecanismo de 
transmisión neuronal se basa en que la concentración de ión $\mathrm{Na}+$ es elevada fuera de la célula y baja dentro de ella, en cambio la concentración de ión $\mathrm{K}+$ es alta en el interior de la célula y baja en el exterior. El potencial de reposo del nervio es de $-90 \mathrm{mV}$, siendo el resultado de una mayor salida de iones $\mathrm{K}+$ que entrada de iones $\mathrm{Na}+$. Este gradiente iónico del sodio y del potasio a través de la membrana celular se mantiene gracias a una bomba traslocadora de iones sodio-potasio ATP-dependiente de la membrana celular. Los anestésicos locales inhiben el paso de los iones $\mathrm{Na}+$ hacia la parte interior de la membrana, sin alterar los movimientos de $\mathrm{K}+$ hacia el exterior. La aplicación de un anestésico local sobre un nervio no modifica el potencial de reposo ni el dintel del potencial de acción ni la repolarización, pero disminuye la velocidad de ascensión de la curva de despolarización, sobre todo en su parte inicial lenta. Los anestésicos locales se depositan en el interior de los canales de sodio después de haber penetrado en el interior de la célula a través de la bicapa lipídica de la membrana celular, es decir, entran en el canal desde la cara interna de la membrana. La velocidad de fijación para el bloqueo fásico, como la velocidad de comienzo para el bloqueo tónico es mayor con un pH exterior alcalino que favorece la forma neutral del fármaco a nivel de la membrana y las formas neutral y catiónica a nivel del citoplasma, motivo por el que los anestésicos locales tienen menor efecto en zonas inflamadas, con pH más ácido que en zonas normales"6. "Impide que se propaguen los impulsos de las terminaciones nerviosas con un agente anestésico que se aplica en las terminaciones nerviosas libres, el nombre tópica indica que solo basta el contacto de la sustancia con la superficie, no se utilizan inyecciones"7. "La anestesia tópica está disponible en tres formas: gel, líquido y spray. Los geles y los líquidos se recomiendan antes de la anestesia local o durante procedimientos de raspado radicular, con la finalidad de prevenir el dolor de tejidos blandos"8. "La Lidocaína Introducida en 1948 es uno de los anestésicos locales que más se usan, pues produce una anestesia más rápida, intensa, duradera y amplia que la procaína y posee unos efectos tópicos muy buenos. Es el agente de elección en pacientes sensibles a los esteres. Se consigue en forma líquida para inyecciones, jalea, crema, ungüentos y aerosol"9. "Nombre comercial: Xilocaĺna Nombre químico: Dietilamino -2,6acetoxilidida. Propiedades: Es muy soluble en agua, puede someterse a ebullición durante 8 horas, se puede reesterilizar en autoclave sin perder su potencia, no irrita los tejidos, incluso a concentraciones de $88 \%$. Es $1,5 \%$ más tóxica que la procaína (tomando como referencia el valor de 1 ) y apenas tiene $20 \%$ de la toxicidad de la cocaína"10.

\section{MATERIALES Y MÉTODOS}

El estudio tuvo un diseño cuasi-experimental, los parches fueron colocados en las hemiarcadas de un mismo paciente, se conformaron dos grupos, donde se planteó la fórmula de muestreo para comparación de medias, la cual determinó un tamaño de muestra de 28 unidades de estudio en cada grupo.

El parche llamado OUTDOL, tuvo como principio activo la lidocaĺna al $20 \%$, denominada químicamente como acetamida, 2(dihetilamino)- $\mathrm{N}$-(2,6-dimetilxilidida), con una fórmula molecular de $\mathrm{C}_{14} \mathrm{H}_{22} \mathrm{~N}_{2} \mathrm{O}$ y el peso molecular es 234.3411-12-13.

La medida de cada parche fue de $5 \mathrm{~cm} \times 1 \mathrm{~cm}$ (Fig.2), con una cantidad de $115.25 \mathrm{mg}$ de lidocaína de $20 \%$ como ingrediente activo en cada uno.

Se colocaron los parches dentro del vaso dapen, quedando humedecidos completamente (Fig.3), luego se procedió a mezclar el adhesivo (polivinil acetato), con digluconato de clorhexidina en gel al $0.12 \%$, en una proporción de 1 a 2, respectivamente (Fig. 4), se formó una pasta uniforme y se untó en cada parche.

Se obtuvieron 28 parches con el principio activo, digluconato de clorhexidina al $0.12 \%$ y adhesivo; 28 parches sin el principio activo, con la finalidad de tener un grupo experimental y grupo control. Luego de fabricar los parches, se dejaron secar en una caja de instrumental estéril, separados de tal manera que solo la investigadora supiera cual era el parche que llevaba el principio activo. Todos los parches estuvieron consignados en la ficha de toma de datos.

Cada parche fue colocado en la hemiarcada superior y se esperó un tiempo de 5 minutos por parche y luego fueron retirados (Fig5) y (Fig6).

Para realizar el procedimiento de raspado supra gingival, se calibraron 4 operadores. El procedimiento de detartraje fue estandarizado, se sostuvo la chuchilla bajo la técnica de pluma modificada y se estableció un apoyo digital firme sobre los dientes vecinos de la zona de trabajo. La hoja del instrumento tuvo con una angulación inferior a 90 grados con respecto a la superficie del diente a raspar. El borde cortante del instrumento estuvo en el margen apical del cálculo supra gingival, se realizaron movimientos cortos y energéticos (Fig.7).

Para comprobar la validez del instrumento EVA (Escala Visual Analógica de dolor con un valor de 5 puntos) se realizó una prueba piloto, con 5 unidades de estudio, los cuales se incluyeron en el estudio. 
Para recopilar los datos, se explicó al paciente el tipo de anestesia y la técnica que se aplicaría; también se informó sobre el parche y la función de este mismo en el procedimiento de detartraje. Antes de realizar el procedimiento se pidió que acepte y firme el consentimiento informado.

Se aplicó el parche OUTDOL en la hemiarcada superior así como también el parche sin el principio activo, se esperó un tiempo de 5 minutos y se retiraron los parches. Se realizó el tratamiento de detartraje en ambas hemiarcadas superiores. Se preguntó al paciente si tuvo alguna molestia o sintió dolor en la encía, debido al instrumento y las respuestas fueron anotadas en las fichas.

\section{RESULTADOS}

La edad de los pacientes se presentó entre los 18 años y los 50 años. El promedio de edad de esta muestra estuvo en 33 años, con una desviación estándar de 12,65. La muestra estudiada presentó un mayor número de personas de sexo femenino. (Gráfico 1)

Los valores de dolor presentados en el grupo control, mostraron valores desde 0.00 hasta 1.43 , en una escala del 0 al 5 , los valores que conglomeraron la mayor cantidad de frecuencia fueron 0.80 y 1.00 como promedio del dolor en las encías de piezas destartarizadas. El promedio de puntaje de dolor del grupo control se presentó en 0.95 , mientras que un $50 \%$ de los valores se agrupan por debajo de 1.00 y el otro $50 \%$ se presenta ente 1.1 hasta 1.43. La desviación estándar de 0.39 nos lleva a verificar que hay un coeficiente de variación de $41 \%$. El coeficiente de asimetría con valor negativo, nos indica que los valores tienden a presentar una cola hacia valores menores.

En el gráfico 2 se puede apreciar que el grupo control mostró mucho más dolor que el grupo experimental, siendo el promedio de dolor 0.28 para el grupo experimental y 0.94 para el grupo control. Los valores de dolor presentados en el grupo experimental, mostraron valores desde 0.00 hasta 1.43 , en una escala del 0 al 5 , los valores que conglomeraron la mayor cantidad de frecuencia fueron 0.14 y 0.29 como promedio del dolor en las encías de piezas destartarizadas de este grupo experimental.

El promedio de puntaje de dolor del grupo control se presentó en 0.29 , mientras que un $50 \%$ de los valores se agrupan por debajo de 0.14 y el otro $50 \%$ se presenta ente 0.15 hasta 1.43 . La moda presenta el valor 0.14 como el más repetido. La desviación estándar de 0.39 nos lleva a verificar que hay un coeficiente de variación de valor alto.

Tabla 1. Comparación de estadísticos del puntaje de dolor. Diferencia de medias -0.66 Valor de T Student Sig $<0.01$

\section{EXPERIMENTAL}

\begin{tabular}{ccc}
\multicolumn{3}{c}{ CONTROL } \\
\hline Media & 0.95 & 0.29 \\
Mediana & 1 & 0.14 \\
Moda & 1 & 0.14 \\
& & \\
Desv. típ. & 0.39 & 0.39 \\
& & 2.49 \\
Asimetría & -1.32 & \\
& & \\
Curtosis & 1.31 & \\
& & \\
\hline
\end{tabular}

La diferencia de promedios de puntaje de dolor ente el grupo experimental y el grupo control, es -0.66 , con una desviación estándar de 0.73. El Valor de T calculado resultó ser -4.28 para 27 grados de libertad, dando un intervalo de confianza al $95 \%$; entre los valores de -0.95 y -0.38 , lo que significa que la diferencia entre los promedios es estadísticamente significativa, lo que se comprueba con el valor $0.00 \mathrm{de}$ la significancia bilateral, siendo < a 0.05 entonces se acepta la hipótesis de diferencia de promedios entre el grupo control y el grupo experimental. (Tabla 1)

Los valores de puntuación del grupo control se presentaron en el rango de 1.43 puntos teniendo como valor mínimo 0.00 y como valor máximo 1.43; el promedio se presentó en 0.95 puntos. En cuanto al grupo experimental, el rango fue de 1.43 puntos, teniendo como valor mínimo 0.00 y como valor máximo 1.43; mientras que el promedio se presentó en 0.29 puntos. 


\section{DISCUSIÓN}

El dolor de la zona sin parche que fue referido por los pacientes de 18 a 50 años de edad del servicio de periodoncia en la clínica Odontológica de la Universidad Católica de Santa María de Arequipa Perú, fue en promedio de 0.95 con desviación estándar de 0.39 , en una escala de 0 a 5 . El dolor en la zona del parche, 0.29 con desviación estándar de 0.39 , por lo que se considera una reducción de $30 \%$, esto se debe a la adhesividad del parche que permite la permanencia del principio activo en contacto con la mucosa por más tiempo que una anestesia tópica, lo que concuerda con los hallazgos en 2011 de Cruz-Antonio, Leticia et al, quienes en la Revista Mexicana de Ciencias Farmaceúticas presenta un estudio de formulación y evaluación de un parche bucal compuesto de propóleo y Echinacea purpurea ${ }^{14}$.

El parche OUTDOL en su composición tiene clorhexidina, de igual forma en la Universidad de Colombia Jean Mario Castro Ruiz ${ }^{15}$, presentó una tesis titulada Diseño de un sistema bioadhesivo de clorhexidina empleando pullulan como matriz para uso en mucosa oral, que demostró la liberación del 100\% de clorexhidina a los 180 minutos. Lo que abre otro espacio para investigar con este parche, respecto a liberación de fármacos post exodoncia o post tratamiento periodontal ${ }^{15}$.

Igual que en otros estudios la utilidad clínica más relevante de este parche vendría a ser la anestesia pre punción en niños y pacientes nerviosos o con experiencias traumáticas previas, otros estudios han demostrado la preferencia de los niños a favor de un parche adhesivo frente al gel anestésico tradicional ${ }^{16-17-18-19}$.

\section{CONCLUSIÓN}

Existe diferencias estadísticamente significativa entre el dolor de la zona con el parche y la zona sin el parche, demostrado por la prueba estadística T de Student $(p<0.01)$. Por lo tanto la formulación del parche OUTDOL es efectiva en la liberación de anestésico e inducción a la anestesia de la mucosa.

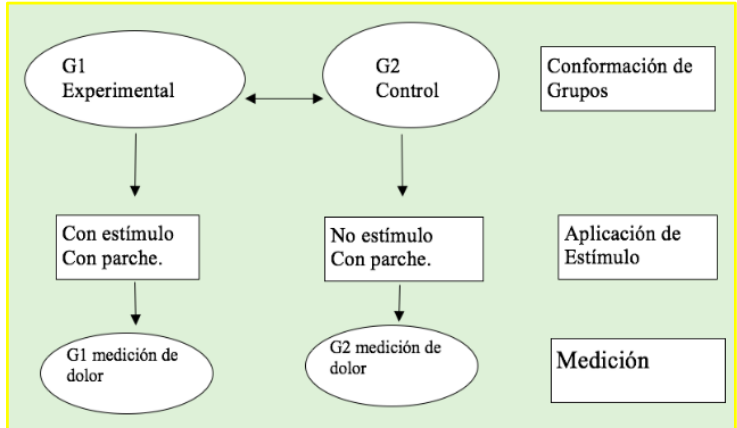

Fig.1 Diseño del estudio

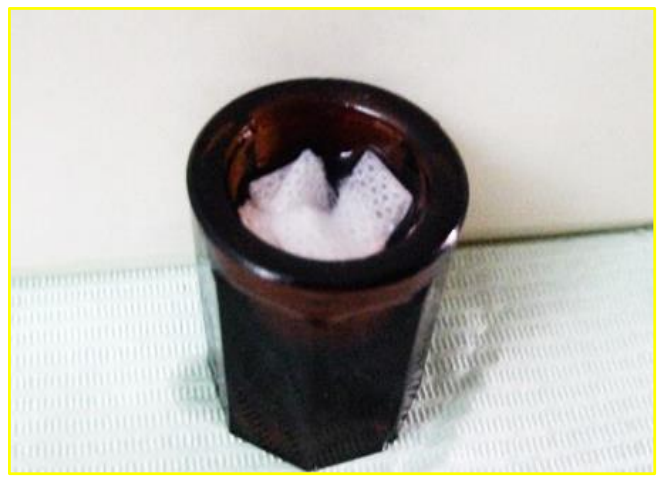

Fig.3 Parches humedecidos con lidocaína
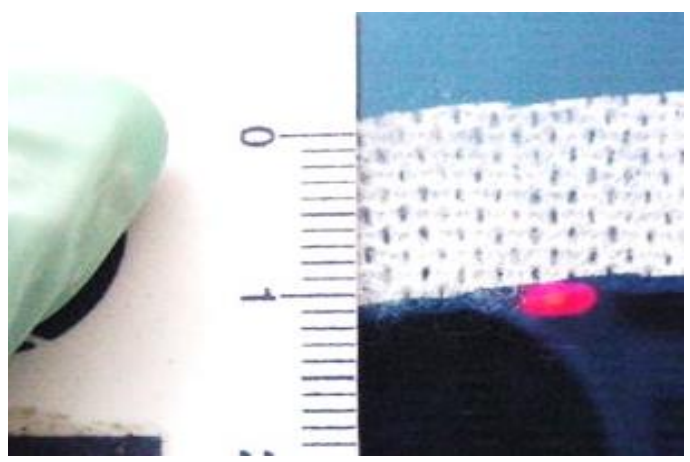

Fig.2 Medida del parche OUTDOL

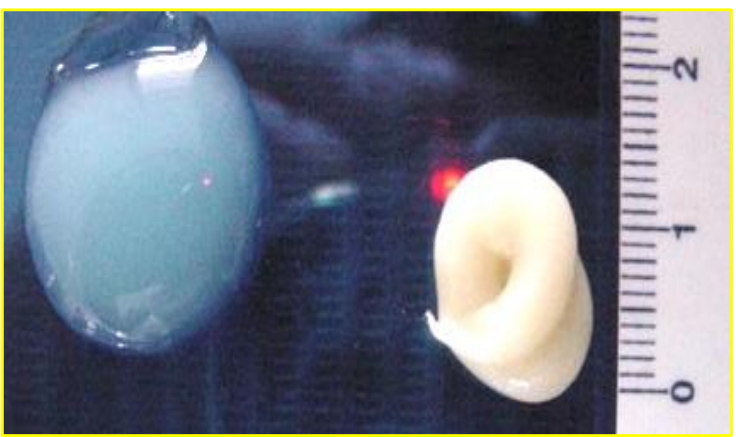

Fig. 4 Dosificación del digluconato de clorhexidina al $0.12 \%$ y el polivinil acetato 


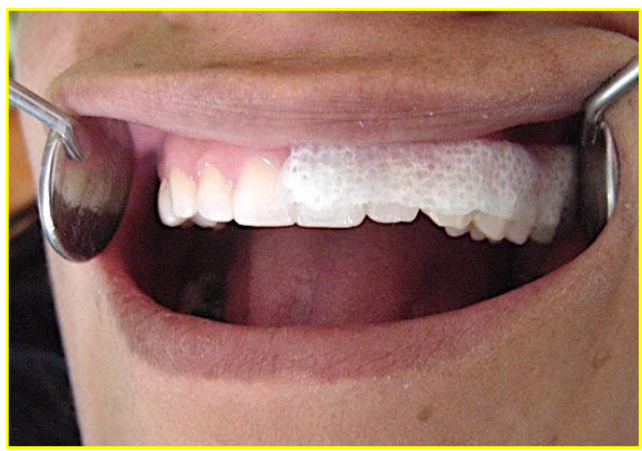

Fig.5 Parche activo OUTDOL en hemiarcada superior.

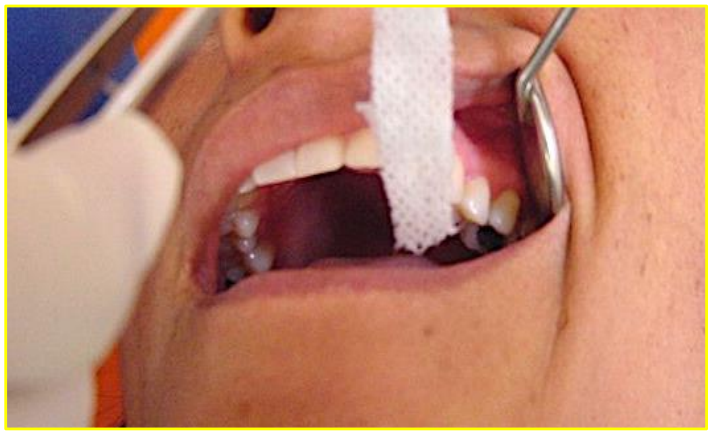

Fig.6 Retiro del parche después de 5 minutos.

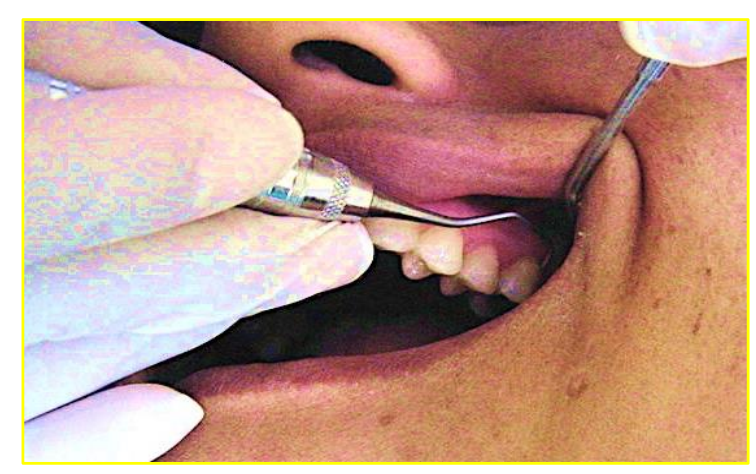

Fig.7 Procedimiento de detartraje.

\section{REFERENCIAS BIBLIOGRÁFICAS}

1. Mueller Hans-Peter. Periodontology. NewYork: Stuttgart;2005.

2. Carranza F. Periodontología. 9 ed. Mc Grawhill.2001

3. Carranza. F.Jr.Periodontologia Clínica. 7 ed. Mc GrawHill.2002

4. Rosado-Linares L. Manual de Periodoncia Clínica. Universidad Católica de Santa María, ArequipaPerú 1993.

5. Bartíos G. Odontología: su fundamento biológico, Volumen 1. Medellín Colombia. latros;1993

6. Carranza S. Compendio de Periodoncia, 5 ed. Editorial Médica Panamericana.

7. Gurrola-Martínez B. Manual de Anestesia Odontológica. Primera ed. Mexico DF: McGraw - Hill Interamericana; 2001.

8. Woodall I. Tratado de Higiene Dental. Tomo II. Madrid. Salvat 1992.

9. Goodman y Gilman, Las Bases Farmacologicas De La Terapeuta. 11 ed. Mc Graw Hill.

10. Macouzet-Olivar C. Anestesia Local en Odontologia. Mexico DF: Manual Moderno; 2008.

11. Leopold A1, Wilson S, Weaver JS, Moursi AM. Pharmacokinetics of lidocaine delivered from a transmucosal patch in children. Anesth Prog. 2002 Summer;49(3):82-7.

12. Leon M.E. Anestésicos locales en odontología. Colombia Médica, Vol.32 N 3 2001, Corporación editora Medica del Valle 2001.

13. Pennington G.W. Farmacología Dental, Noriega Editores 1984.

14. Cruz-Antonio L, Sánchez-Mendoza ME, Guillén E, Vidal MA, Arrieta J. Formulación y evaluación de un parche bucal compuesto de propóleo y Echinacea purpurea Revista Mexicana de Ciencias 
Farmacéuticas, vol. 42, núm. 4, octubre-diciembre, 2011, pp. 50-56 Asociación Farmacéutica Mexicana, A.C. Distrito Federal, México.

15. Castro-Ruiz JM. Diseño de un sistema bioadhesivo de clorhexidina empleando pullulan como matriz para uso en mucosa oral Diseño de un sistema bioadhesivo de clorhexidina empleando pullulan como matriz para uso en mucosa oral. Tesis de titulación. Bogotá D.C., Colombia: Universidad Nacional de Colombia, Facultad de ciencias, Área curricular de Farmacia; 2014.

16. Stecker r SS, Swift JQ, Hodges JS, Erickson PR. Should a mucoadhesive patch (DentiPatch) be used for gingival anesthesia in children? Anesth Prog. 2002 Winter;49(1):3-8

17. Primosch RE, Rolland-Asensi G. Comparison of topical EMLA 5\% oral adhesive to benzocaine $20 \%$ on the pain experienced during palatal anesthetic infiltration in children. Pediatr Dent. 2001 JanFeb;23(1):11-4. PubMed PMID: 11242723.

18. Kreider KA, Stratmann RG, Milano M, Agostini FG, Munsell M. Reducing children's injection pain: lidocaine patches versus topical benzocaine gel.Pediatr Dent. 2001 Jan-Feb;23(1):19-23. PubMed PMID: 11242725.

19. Wu SJ, Julliard K. Children's preference of benzocaine gel versus the lidocaine patch. Pediatr Dent. 2003 Jul-Aug;25(4):401-5. PubMed PMID: 13678108. 